\title{
Real-Time Path Planning for Time-Optimal Exploration
}

\author{
Andrew T. Klesh, Anouck R. Girard†, and Pierre T. Kabamba ${ }^{\ddagger}$ \\ University of Michigan, Ann Arbor, Michigan, 48109
}

\begin{abstract}
Motivated by cooperative exploration missions, this paper considers constant velocity, level flight path planning for Unmanned Air Vehicles (UAVs) equipped with range limited, omni-directional sensors. These active energy-based sensors collect information about objects of interest at rates that depend on the range to the objects according to Shannon's channel capacity equation, where the signal to noise ratio is governed by the radar equation. The mission of the UAVs is to travel through a given area and collect a specified amount of information about each object of interest while minimizing the total flight time. This information can then be used to classify the objects of interest. An optimal path planning problem is examined where the states are the coordinates of the UAVs and the amounts of information collected about each object of interest, the control inputs are the UAV heading angles, the objective function is the elapsed time, and the boundary conditions are subject to inequality constraints that reflect the requirements of information collection. Based on this problem, several technical approaches are suggested for the implementation of a real-time path planner. The computational speed of the path planner is dependent upon onboard processing capability. As an optimal solution may not be feasible during an allowed planning interval, the problem becomes one of bounded rationality, where the optimality of the solution is directly dependent upon the length of computational time available. A parametric approximation and table lookup strategy are discussed as two possible methods of approaching bounded rationality. These strategies are implemented in a receding horizon controller suitable for a time-varying environment. The methods are compared by their various virtues and the results are illustrated on several time-optimal exploration scenarios.
\end{abstract}

\section{INTRODUCTION}

\section{I.A. Overview}

This paper is devoted to the problem of planning the paths of vehicles for exploration of a given area. By exploration we mean collecting information about objects of interest with known locations, where information is understood in the classical sense of Shannon ${ }^{20}$ as a "selection from a set." Although the information collected is to be used to classify the objects of interest (e.g., friend or foe, interesting or uninteresting, etc.), this paper focuses on information collection rather than classification. In this paper, information is collected by active onboard omni-directional sensors that read energy signals reflected off the objects of interest (e.g., radar, sonar), implying a signal-to-noise ratio that decays as the reciprocal of the fourth power of the range.

The key idea of this work is to recognize and exploit the similarity between communication and exploration. Specifically, exploration can be viewed as a communication process where the object of interest is the transmitter, the sensor is the receiver, the sensing processes is the noisy communication channel, and the sensed signal carries information about the object of interest. Consequently, the maximum rate at which

${ }^{*}$ A. Klesh is a PhD Candidate, Aerospace Engineering, University of Michigan, 1320 Beal Ave, Ann Arbor, MI USA aklesh@umich.edu

${ }^{\dagger}$ A. Girard is an Assistant Professor, Aerospace Engineering, University of Michigan, 1320 Beal Ave, Ann Arbor, MI USA anouck@umich.edu

${ }^{\ddagger} \mathrm{P}$. Kabamba is a Professor, Aerospace Engineering, University of Michigan, 1320 Beal Ave, Ann Arbor, MI USA kabamba@umich.edu 
the sensor can collect information about the object of interest is in fact the capacity ${ }^{20}$ and depends on the signal-to-noise ratio of the channel through Shannon's equation.

Hence, the problem of exploration features the coupling between two subsystems - vehicle kinematics and information collection - where the coupling occurs through the signal-to-noise ratio of the sensor. Accounting for this coupling by a practical controller in the design of optimal paths for exploration is the main conceptual contribution of this paper.

\section{I.B. Motivation}

Unmanned Air Vehicles (UAVs) are increasingly used for dirty, dull or dangerous missions. ${ }^{16}$ The most common use of UAVs is the collection of data for Intelligence, Surveillance and Reconnaissance missions. We are particularly interested in missions where UAVs are tasked with exploring a given area. An example of such a mission involves Mars exploration where objects of interest have been located by the Mars Global Surveyor. UAVs can investigate these objects, but must plan flight paths to assure the collection of the specified amount of information. Moreover, it is often the case that gathering information quickly is of paramount importance (e.g., for tactical reasons in war or to avoid the rapid onset of inclement weather). Because the processors available onboard UAVs are typically slow, the problem becomes one of bounded rationality, that is, having to choose a near-optimal path because of the time constraints. This motivates the techniques presented in this paper.

\section{I.C. Literature Review}

A large body of research has been published in recent years about motion and real-time control of autonomous vehicles. Although an exhaustive overview of the state of the art is beyond the scope of this paper, a brief review of the most relevant literature is as follows.

Many methods exist for solving the basic trajectory-planning problem. ${ }^{11}$ However, not all of them solve the problem in its full generality. For instance, some methods require the workspace to be two-dimensional and the obstacles, if any, to be polygonal. Despite many external differences, the methods are based on few different general approaches: roadmap, ${ }^{11,13,25}$ cell decomposition, ${ }^{14,19}$ potential field ${ }^{1,9}$ and probabilistic. ${ }^{6,23}$ Optimal control approaches have also been studied..$^{3,22}$

Receding horizon control, introduced in the early 1980 's,${ }^{10}$ has been utilized to control linear and nonlinear systems. ${ }^{15}$ Recent advances in onboard processing have allowed implementation on $\mathrm{UAVs}^{7}$ for obstacle avoidance and path planning. Such real-time processing permits more complicated path-planning strategies. Here we define real-time processing as computation with a total duration short compared to the duration of the flight path of the UAV. To date, real-time receding horizon control has not been coupled with information based path planning.

Other real-time control strategies, such as table lookup, have also been implemented on UAVs. ${ }^{2,17}$ Table lookup requires little processing power but a large amount of onboard storage. Today, storage is very cheap and volumetrically small providing the ability to store high fidelity tables. Few path planning strategies have taken advantage of this method.

Cooperative vehicles collecting information in an optimal manner has been considered in several papers. ${ }^{4,24}$ Although the current literature discusses various methods of planning optimal paths for UAVs, no real-time implementation accounts for the coupling between UAV kinematics and information collection through the signal-to-noise ratio of the sensors. The current paper addresses this issue.

\section{I.D. Original Contributions}

This paper analyzes an integrated model of the aircraft kinematics and information collection, applicable to cooperative exploration, with the following features. First, the rate at which a sensor collects information about an object of interest is specified by Shannon's channel capacity equation, ${ }^{20}$ which depends on the sensor signal-to-noise ratio. Second, the signal-to-noise ratio decays as the reciprocal of the fourth power of the range to the object of interest, according to the radar equation. ${ }^{5}$ Hence, this paper accounts for the coupling between vehicle kinematics and information collection.

Based on the integrated model, the problem of exploration for UAVs is formulated as an optimal path planning problem where the states are the coordinates of the UAVs and the amounts of information collected about each object of interest, the control inputs are the UAV heading angles, the objective function is the 
elapsed time, and the boundary conditions are subject to inequality constraints that reflect the requirements of information collection. This optimal path planning problem can be solved in real-time if treated as a bounded rationality problem. The present paper studies this rationality problem and provides the following original contributions:

- Parametric approximation and table lookup strategies are applied to address bounded rationality.

- The two strategies are compared against an optimal solution by storage and computation requirements.

- Receding horizon control is used to address combinatorial scheduling.

\section{I.E. Paper Outline}

The remainder of the paper is as follows. In Section II, the integrated model is presented. In Section III, the optimization problem is formulated to minimize the mission time of the UAVs. Section IV presents the numerical procedure used to generate optimal paths and the method used to perform a parametric study. Section V presents strategies to address bounded rationality and Section VI a receding horizon controller. Example solutions from the real-time planners are illustrated in Section VII, while conclusions and future work are discussed in Section VIII. In Appendix A, the model and problem are non-dimensionalized for scaling purposes.

\section{Modeling}

The model considered in our problem formulation consists of two parts: the aircraft kinematic model and the information collection model.

\section{II.A. The Aircraft Kinematic Model}

The aircraft kinematic model is based upon the unicycle vehicle model: ${ }^{18}$

$$
\begin{aligned}
X_{i}^{\prime} & =V \cos \psi_{i}, 1 \leq i \leq n, \\
Y_{i}^{\prime} & =V \sin \psi_{i}, 1 \leq i \leq n,
\end{aligned}
$$

where $X_{i}$ and $Y_{i}$ are the Cartesian coordinates of the $i$ th aircraft, prime denotes time derivative, $V$ is the velocity of the aircraft, $\psi_{i}$ is the heading of the $i$ th aircraft and $n$ is the number of aircraft. For simplicity, $V$ is assumed to be the same for all aircraft.

\section{II.B. The Information Collection Model}

We seek to explore a given area, by which we mean to collect a specified amount of information about each of $m$ objects of interest, at known locations in the area. Without loss of generality, assume that the required amount of information is one bit for each object. To collect information, we use onboard active, energy-based sensors, e.g., radar.

The key idea of our work is to recognize and exploit the similarity between communication and exploration. Specifically, exploration can be viewed as a communication process where each object of interest is a transmitter, the explorer is the receiver, the sensing processes are noisy communication channels, and the sensor signals carry information that allows the explorer to identify the objects of interest.

Now the maximum rate at which information can be transmitted over a noisy communication channel (i.e., the channel capacity) is, ${ }^{20}$

$$
\dot{I}=W \log _{2}(1+\mathrm{SNR}),
$$

where $W$ is the channel bandwidth and SNR is the signal-to-noise ratio.

Moreover, a radar sensor located at Cartesian coordinates $(X, Y)$ and observing an object at Cartesian coordinates $(A, B)$ will provide a reading with signal-to-noise ratio of the form, $:^{5}$

$$
\mathrm{SNR}=\frac{K^{4}}{\left((X-A)^{2}+(Y-B)^{2}\right)^{2}},
$$


where the parameter $K$ depends on the object and represents its visibility.

Combining (3) and (4), the information collection model is as follows. Here we assume that information is additive. Let $I_{j}$ denote the amount of information that the $n$ aircraft have cooperatively collected about the $j$ th object of interest, $1 \leq j \leq m$. Then,

$$
\dot{I}_{j}=W_{i} \sum_{i=1}^{n} \log _{2}\left(1+\frac{K_{j}^{4}}{\left(\left(X_{i}-A_{j}\right)^{2}+\left(Y_{i}-B_{j}\right)^{2}\right)^{2}}\right), 1 \leq j \leq m,
$$

where we assume that all radar sensing processes, viewed as communication channels, have the same bandwidth, and the parameters $K_{j}$ depends on the $j$ th object of interest, which is located at Cartesian coordinates $\left(A_{j}, B_{j}\right)$.

When the vehicle is close to the $j$ th object and can collect information at an appreciable rate, the object is considered visible and the vehicle is within that object's visibility disc. Otherwise the object is invisible to the vehicle. Objects are said to be clustered if their visibility discs are pathwise connected. Furthermore, clusters are said to be isolated if they are not pathwise connected. Finally, an object is said to be isolated if it is not in any cluster.

\section{Problem Formulation}

The problem treated in this paper is to minimize the total flight time required for $n$ UAVs to collect a specified amount of information about $m$ objects of interest in a given area. The UAVs under consideration have active energy-based sensors and have enough fuel to complete a mission with constant altitude flight. It is assumed that each UAV has been assined apriori a number of objects of interest to visit. Thus, the real-time controllers discussed in this paper are constructed for a single UAV to plan its individual path.

The objects of interest are assumed isotropic in that all relative bearings yield the same rate of information collection. We assume no redundant information is useful, i.e., only one bit of information about each object of interest is needed to accomplish the mission. Since the information is collected at the most optimistic rate possible, (5) becomes a lower bound on any rate of information collection. A conservative upper bound is found in Ref. 18. Future work will consider reducing this upper bound based on our new information collection model.

The aircraft begins at a given location with a free heading and must collect at least one bit of information about each object in the area. Active sensors, based upon (5), are employed by the aircraft to collect information. There is no onboard storage limit nor processing requirement for the acquisition of information.

In a real mission, the computation time available for deriving an optimal path is often limited by external events (e.g. inclement weather, strategic decisions, etc.). This leads to a bounded-rationality problem, ${ }^{21}$ where the performance of a given solution is a function of the time allowed to solve the problem. As more time is provided, the performance increases, and, if infinite time was given to solve the problem, a optimal solution would be found. As this is not practical, we seek to implement strategies to provide near-optimal solutions, where the performance depends on the bound on rationality.

The UAVs under consideration fly within a time-varying environment and changes to the flight path can occur often. We implement a receding horizon controller to address the complexity induced by changes. Specifically, rather than plan a path between every object in the exploration area, we seek only to plan an optimal path between the next two objects (the planning horizon) and plan the next segment of the path once an object is reached. This allows for a changing environment, where objects might alter their position, while still providing near-optimal paths.

The dynamic optimization problem presented in this paper is motivated by the requirement to minimize, with respect to the time-history of the heading angles, the final time of mission, i.e.,

$$
\min _{\psi_{i}(\cdot)} t_{f}
$$


subject to (1)-(5) and boundary conditions:

$$
\begin{gathered}
X_{i}(0) \text { given, } 1 \leq i \leq n, \\
Y_{i}(0) \text { given, } 1 \leq i \leq n, \\
I_{j}(0)=0,1 \leq j \leq m, \\
X_{i}\left(t_{f}\right) \text { free, } 1 \leq i \leq n, \\
Y_{i}\left(t_{f}\right) \text { free, } 1 \leq i \leq n, \\
I_{j}\left(t_{f}\right) \geq 1,1 \leq j \leq m .
\end{gathered}
$$

\section{The Discretization Procedure}

To obtain numerical approximations of optimal paths, we discretize the problem as follows. For a chosen integer $p \geq 1$, we subdivide the interval $\left[t_{o}, t_{f}\right]$ into $p$ subintervals $\left[t_{o}, t_{1}\right],\left[t_{1}, t_{2}\right], \ldots,\left[t_{p-1}, t_{f}\right]$ of equal duration. In each subinterval we assume that the control inputs are constant, i.e., $\left(\psi_{i}(t)\right)=\left(\psi_{i_{g}}\right), t \in\left[t_{g}, t_{g+1}\right]$, where the parameters $\psi_{i_{g}}, 0 \leq g \leq p-1$, are unknown.

We treat the parameters $\psi_{i_{q}}, 0 \leq g \leq p-1$, and $t_{f}$ as inputs to a nonlinear optimization problem. As an initial choice, in all subintervals we choose $\psi_{i_{g}}=0$ and $t_{f}=t_{o}+T_{M}$ where $T_{M}$ is a maximum duration allowed. Constraints upon this problem are imposed from the boundary conditions (7)-(12). From (6), the objective function is the total flight time. We then numerically solve for optimal flight paths using the MATLAB ${ }^{\circledR}$ Optimization Toolbox function fmincon and the ordinary differential equation solver ode 45 . We will call this strategy the discretization method.

\section{Bounded Rationality}

When a dynamically replanning in changing environments, course corrections must be made quickly. UAV processors are ill-equipped to solve the optimal path planning problem in real-time. Instead, this problem becomes one of bounded rationality. Two strategies for finding near-optimal paths with bounded rationality are discussed in this section: parametric study and table-lookup.

\section{V.A. The Parametric Study}

Several properties of optimal paths amongst isolated objects are discussed in 8 and summarized below:

Proposition 1:If the objects of interest are isolated, then the optimal flight paths consist of sequences of straight lines (far from the objects of interest) connected by short turns (near the objects of interest).

Corollary 1:If in addition to being isolated, the objects of interest are poorly visible, then the problem becomes a Multi-vehicle Traveling Salesperson Problem (MTSP). ${ }^{12}$

Proposition 1 and Corollary 1 provide a basic model of the optimal paths amongst several objects of interest. We seek to find a higher fidelity model describing the optimal flight paths implementable onboard an aircraft. Without loss of generality, we can use a small set of objects of interest to examine properties of the optimal paths. An example path amongst two objects of interest with one UAV is shown in Figure 1. By gradually varying individual parameters, we can generate many solutions and optimal path properties can be identified. A list of the possible parameters to be varied is shown in Table 1. Note: Lowercase variables imply scaling is included (See Appendix A).

Expressions can be formed describing optimal paths by varying the parameters in table 1 and recording characteristics about the paths, using the varied parameter as the independent variable.

As an example, the visibility of the first object, $k_{1}$, is varied from small to large. The resulting flight path solutions have been plotted in figure 2. As predicted in Proposition 1, every flight path is made up of straight lines and short curves. As $k_{1}$ is increased, the location where the UAV passes closest to the first object of interest increases as well. This distance can be plotted as a function of $k_{1}$ as shown in figure 3 and a trendline, (13), can be associated with the plot.

$$
\text { Minimum Approach Distance }=0.00005\left(k_{1} w t_{c} / l_{c}\right)^{2.8898}
$$




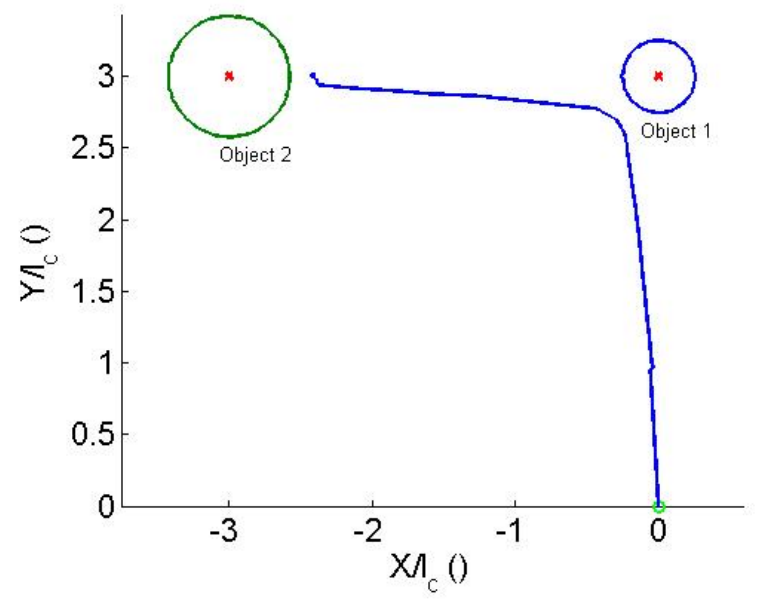

Figure 1. An example flight path with a single UAV and two objects of interest created with the discretization method

Table 1. Parameters varied in the parametric study

\begin{tabular}{ll}
\hline$V$ & Velocity of the aircraft, $(\mathrm{m} / \mathrm{s})$ \\
$K_{1}$ & Visibility of the first object $(\mathrm{m})$ \\
$K_{2}$ & Visibility of the second object $(\mathrm{m})$ \\
$W$ & Bandwidth of the onboard sensor $(\mathrm{bits} / \mathrm{sec})$ \\
$\left(A_{1}, B_{1}\right)$ & Location of the first object $(\mathrm{m}, \mathrm{m})$ \\
$\left(A_{2}, B_{2}\right)$ & $\begin{array}{l}\text { Location of the second object }(\mathrm{m}, \mathrm{m}) \\
\theta\end{array}$ \\
& $\begin{array}{l}\text { Relative angle between the initial } \\
\text { location of the UAV and the line of sight first } \\
\text { object and the line of sight from the first object } \\
\text { to the second object }(\mathrm{deg})\end{array}$ \\
\end{tabular}

Similar sets of solutions can be found for each of the other parameters expressed in table 1 . These solutions experimentally verify Proposition 1 and parameterize flight paths between two objects of interest.

The expression to characterize the minimum approach distance, $r_{1}$, to the first object of interest is:

$$
f_{r_{1}}\left(v, \theta, k_{1} w, I\left(t_{f}\right)\right)=\kappa_{r_{1}}(v) \kappa_{r_{1}}(\theta) \kappa_{r_{1}}\left(k_{1} w\right) \kappa_{r_{1}}\left(I\left(t_{f}\right)\right) R_{1},
$$

where,

$$
\begin{aligned}
\kappa_{r_{1}}(v) & =\left(\frac{1}{-2.08 v^{4}+4.10 v^{3}-0.45 v^{2}+2.85 v+0.25}\right) / R_{1}, \\
\kappa_{r_{1}}(\theta) & =(-0.2597 \theta+0.869) / R_{1}, \\
\kappa_{r_{1}}\left(k_{1}\right) & =\left(0.75 k_{1}^{3}+0.20 k_{1}^{2}-0.084 k_{1}\right) / R_{1}, \\
\kappa_{r_{1}}\left(I\left(t_{f}\right)\right) & =\left(\frac{1}{0.17 I\left(t_{f}\right)^{3}-0.48 I\left(t_{f}\right)^{2}+1.52 I\left(t_{f}\right)-0.04}\right) / R_{1} .
\end{aligned}
$$

and where $R_{1}$ is the nominal minimum approach distance at $v=v_{0}, \theta=\theta_{0}, k_{1}=k_{1_{0}}$ and $I_{t_{f}}=I_{t_{f_{0}}}$.

Similarly, the expression to characterize the radius of the osculating circle, $r_{2}$, that fits the curve near the first object of interest is:

$$
f_{r_{2}}\left(v, \theta, k_{1} w, I\left(t_{f}\right)\right)=\kappa_{r_{2}}(v) \kappa_{r_{2}}(\theta) \kappa_{r_{2}}\left(k_{1} w\right) \kappa_{r_{2}}\left(I\left(t_{f}\right)\right) R_{2}
$$




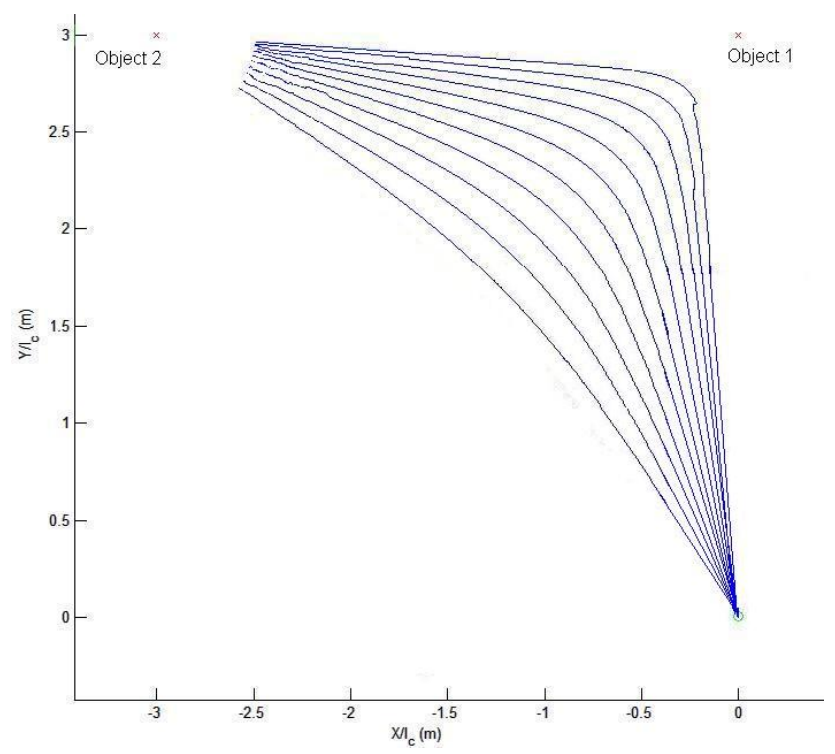

Figure 2. Example flight paths with a single UAV and two objects of interest created when varying $k_{1}$, the visibility of object one

where,

$$
\begin{aligned}
\kappa_{r_{2}}(v) & =\left(\frac{1}{-73.80 v^{4}+54.04 v^{3}-13.8 v^{2}+4.75 v-0.23}\right) / R_{2}, \\
\kappa_{r_{2}}(\theta) & =\left(4.96 \theta^{4}-9.33 \theta^{3}+7.43 \theta^{2}-2.14 \theta+0.31\right) / R_{2}, \\
\kappa_{r_{2}}\left(k_{1}\right) & =\left(2.34 k_{1}^{3}-1.99 k_{1}^{2}+0.88 k_{1}-0.01\right) / R_{2}, \\
\kappa_{r_{2}}\left(I\left(t_{f}\right)\right) & =\left(\frac{1}{-0.06 I\left(t_{f}\right)^{2}+0.87 I\left(t_{f}\right)-0.02}\right) / R_{2} .
\end{aligned}
$$

and where $R_{2}$ is the nominal minimum approach distance at $v=v_{0}, \theta=\theta_{0}, k_{1}=k_{1_{0}}$ and $I_{t_{f}}=I_{t_{f_{0}}}$.

The resulting figuress 4, 5, 2, and 6, and parametric equations (15), (16), (17), (18), (20), (21), (22), and (23) respectively.

\section{V.B. Table-Lookup}

An alternative method to large amounts of computation for in-flight path planning is to precompute some possible flight paths and store them onboard. While this has been impractical in the past, the low cost and volumetric size of memory at the present time allows for large amounts of onboard storage. Here we present a method to utilize a lookup table to form near-optimal paths for the path planning problem discussed in this paper.

The lookup table can be viewed as a $r$-dimensional array, where $r$ is the number of parameters that characterize the table. As in the parametric study, we will consider a four-dimensional space, parameterized by $\left\{v, \theta, k_{1} w, I_{t_{f}}\right\}$. The size of the lookup table across each dimension is a function of the fidelity of the parameters. Indeed, the bound on rationality is implemented through the coarseness of the table. Each entry of the table is the record of a complete path from initial UAV location through two objects of interest based upon the discretization method. As the number of sub-intervals, $p$, in the discretization method increases, the onboard storage requirements of the table will also increase. A three dimensional example of this table is shown in figure 7 .

It should be noted that this problem is not always well-posed. In fact, there are several locations in the parameter-space that are ill-posed, that is, a small variation in the input parameters causes large variations in the optimal flight paths. In general, however, these locations are small.

The total size of the table also determines the offline computational time that is required to build the table. Without enough processing power available, the offline time can be non-trivial. As an example for a lookup table with $r=4$ and $p=20$, the total pre-computational time was seven minutes and the storage 


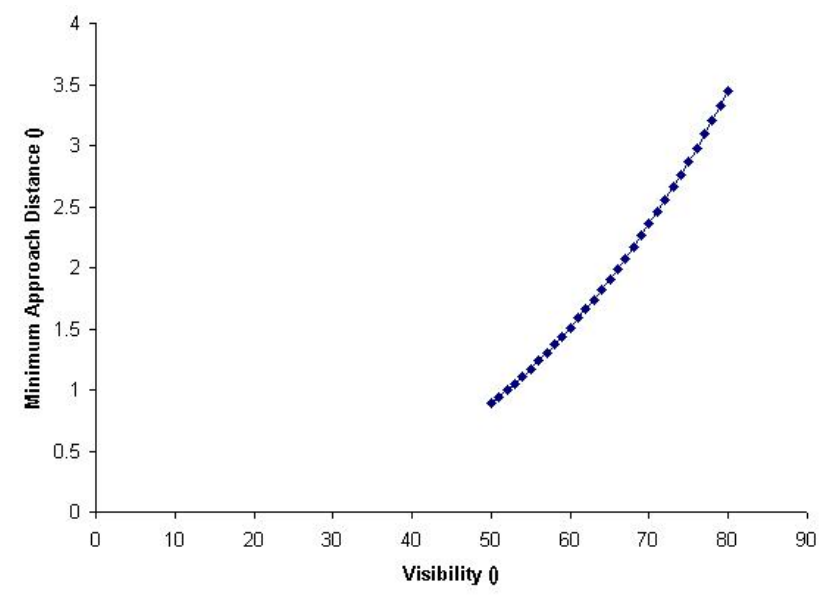

Figure 3. Minimum approach distance of the UAV to object of interest one as a function of visibility

requirements (as found for a Matlab .mat file) is 12.28 Kilobytes. With easily available solid-state memory in 4 Gigabyte packages, the offline computational time is approximately 46 days.

When using the lookup table method, a situation arises quite often where the input parameters do not exactly match entries in the table. In this case, a table entry can be approximated through the use of interpolation between neighboring entries.

\section{Receding Horizon Controller}

A Fixed Horizon Controller ${ }^{10}$ plans a full path in $N$ steps. Each step represents one flight path segment, typically from one object of interest to the subsequent. If the environment changes (an object moves, an obstruction occurs, etc.) this controller is unable to react unless it replans the entire path taking into account the new state information.

Here we consider a Receding Horizon Controller. This controller only plans an optimal path between a limited set of objects, constrained by the so-called planning horizon, a finite number of steps away. After taking a step forward, reaching the execution horizon, the controller replans the path for the next limited set of objects. By stitching together these optimal paths, forward progress is made. If the number of objects considered in the limited set is small, the path planner can quickly react to environmental changes. In this paper, we consider a receding horizon controller with a planning horizon of two objects.

By considering only two objects for our planning horizon, we are able to utilize either strategy previously discussed to quickly calculate our paths. The parametric study provides the minimum approach distance to the first object of interest and the size of the arc of circle the UAV will fly around. We can then construct a near-optimal path from straight lines and arcs of circles. Alternatively, the table lookup method provides a complete optimal path, from which we may have slightly perturbed initial conditions. While the discretization method computes optimal paths on the order of minutes to hours, the receding horizon controller can compute a near-optimal path in milliseconds to seconds.

\section{Illustrations}

Figure 8 presents an optimal flight path for a single UAV exploring an area with two objects of interest. Each object is designated by an $\mathrm{x}$ on the figure. In these examples, it must be noted that the UAV does not fly over the objects of interest, but only approaches each and then turns away towards the next. Thus, the total optimal path consists of straight flights and short turns, as predicted in the proposition and corollary.

The differences in figures 8 and 9 are shown in table 2 . 


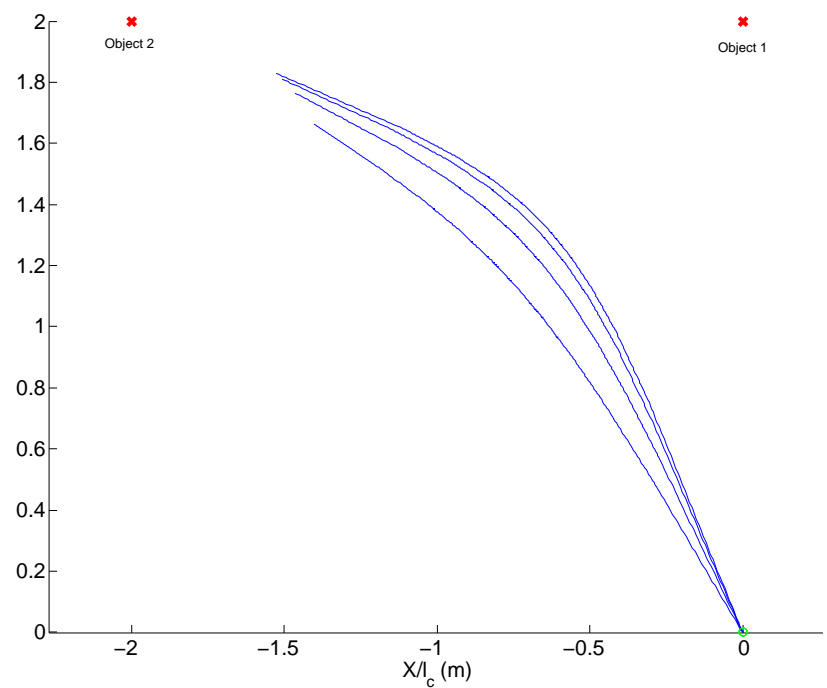

Figure 4. Example flight paths with a single UAV and two objects of interest created when varying $V$, the velocity of the UAV

Table 2. Comparison of the Discretization and Parametric Study Method

\begin{tabular}{lcc}
\hline & Discretization & Parametric Study \\
Computational Time (sec) & 420 & $<1$ \\
Duration of Flight (sec) & 10.627 & 10.7 \\
Information Collected about Object One (bits) & 1 & 1.02 \\
\hline
\end{tabular}

VIII. Conclusions

This paper has presented methods to implement a new information-based formulation for optimal exploration. This formulation exploits the similarity between communication and exploration. We have presented a parametric study analyzing characteristics of optimal paths with multiple objects of interest. The problem of optimal path planning is presented in terms of Shannon's communication theory, the radar equation and aircraft kinematics. We have also implemented a table-lookup planner and examined its relative merits. These methods address bounded rationality have been used to implement a receding horizon controller. These strategies show near-optimal properties in our examples and provide simplifications to the numerical solution. This work is extensible to cooperative exploration with the assumption that the information rates are additive.

In future work, these methods will be rigorously tested on physical autonomous systems and multiple or heterogenous aircraft planners will be considered. The methods presented here are easily extendable to these situations.

\section{Acknowledgements}

This work was supported in part by the United States Air Force under grant number FA 8650-07-2-3744. 


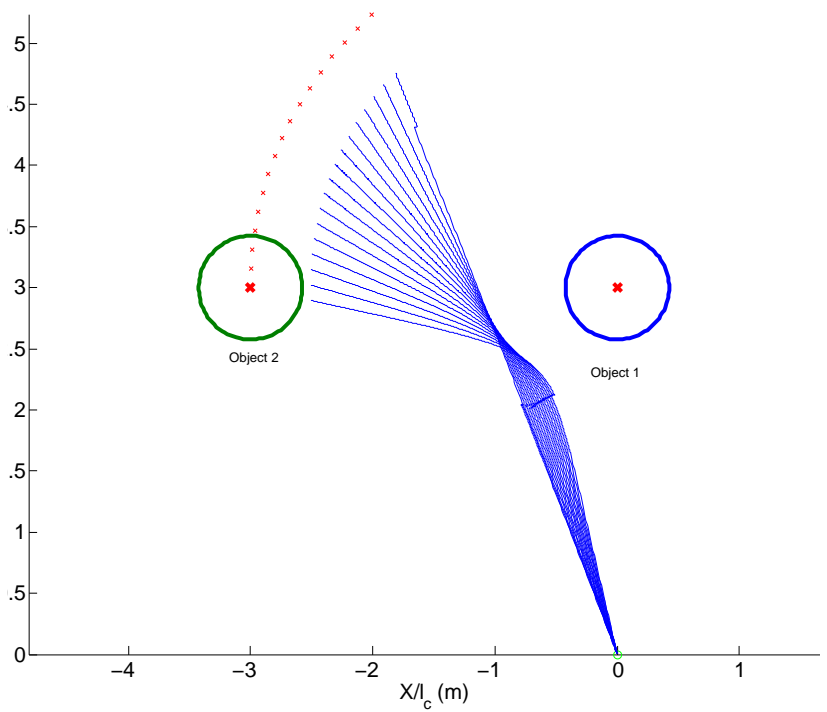

Figure 5. Example flight paths with a single UAV and two objects of interest created when varying $\theta$, the relative angle between the initial location of the UAV and the two objects

\section{Appendix A: Scaling}

If we assume that $l_{c}$ and $t_{c}$ are a characteristic length and time, respectively, we can rewrite (1) - (5) in nondimensional form. We begin by replacing $X_{i}=l_{c} x_{i}, Y_{i}=l_{c} y_{i}$ and $t=t_{c} \tau$ where $x_{i}, y_{i}$, and $\tau$ are nondimensional. Equations (1) - (5) become:

$$
\begin{aligned}
\dot{x}_{i} & =v \cos \psi_{i}, \\
\dot{y}_{i} & =v \sin \psi_{i}, \\
\dot{I}_{j} & =w_{i} \log _{2}\left(1+\frac{k_{j}^{4}}{\left(\left(x_{i}-a_{j}\right)^{2}+\left(y_{i}-b_{j}\right)^{2}\right)^{2}}\right), 1 \leq j \leq m,
\end{aligned}
$$

where the dot denotes derivatives with respect to nondimensional time, $v=\frac{t_{c}}{l_{c}} V, w_{i}=W_{i} t_{c}, k_{j}=\frac{K_{j}}{l_{c}}$, $a_{j}=\frac{A_{j}}{l_{c}}$ and $b_{j}=\frac{B_{j}}{l_{c}}$.

\section{References}

${ }^{1}$ J. Barraquand and J. C. Latombe. Robot motion planning: A distributed representation approach. International Journal of Robotics Research, 10:628-649, 1991.

${ }^{2}$ A Chaudhry, K Misovec, and R D'Andrea. Low observatibility path planning for an unmanned air vehicle using mixed integer linear programming. In 43rd IEEE Conference on Decision and Control, Dec 2004.

${ }^{3}$ L. E. Dubins. On curves of minimal length with a constraint on average curvature, and with prescribed initial and terminal positions and tangents. American Journal of Mathematics, 79:497-517, 1957.

${ }^{4}$ B. Grocholsky, A. Makarenko, and H. Durrant-Whyte. Information-theoretic coordinated control of multiple sensor platforms. Robotics and Automation, 2003. Proceedings. ICRA'03. IEEE International Conference on, 1, 2003.

${ }^{5}$ Pierre Kabamba, S. Meerkov, and Fred Zeitz. Optimal Path Planning for Unmanned Combat Aerial Vehicles to Defeat Radar Tracking. Journal of Guidance, Control and Dynamics, 29:279-288, 2006. 


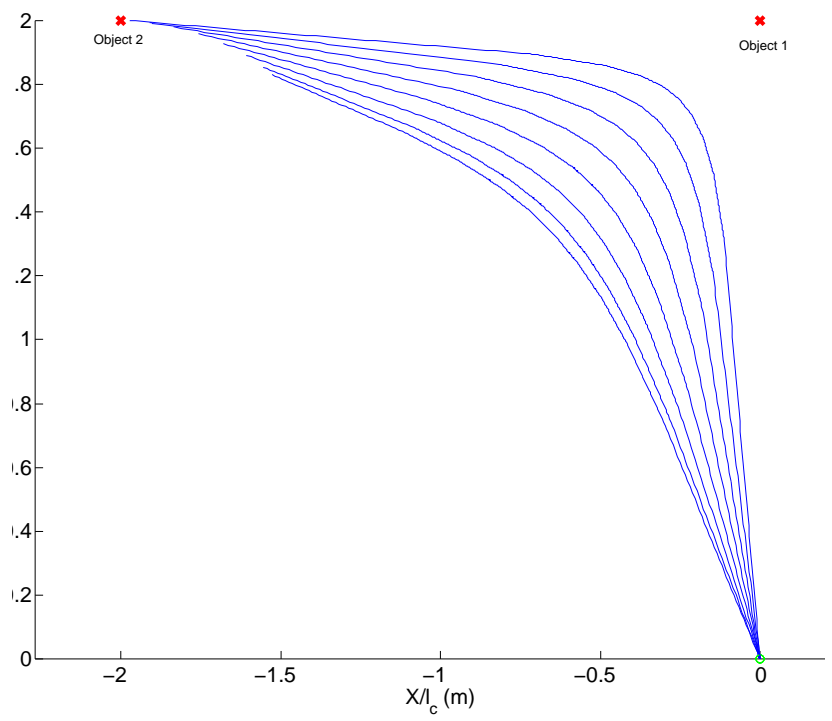

Figure 6. Example flight paths with a single UAV and two objects of interest created when varying $I_{t_{f}}$, the specified amount of information to collect

${ }^{6}$ L. E. Kavraki, P. Latombe, and M. Overmars. Probabilistic roadmaps for path planning in high-dimensional configuration spaces. IEEE Transactions on Robotics and Automation, 12:566-580, 1996.

${ }^{7} \mathrm{~T}$ Keviczky and T Balas. Software-enabled receding horizon control for autonomous uav guidance. In AIAA Guidance, Navigation and Control Conference Proceedings, Aug 2005.

${ }^{8}$ Andrew Klesh, Anouck Girard, and Pierre Kabamba. Path planning for cooperative time-optimal information collection. In 2008 American Controls Conference, Jun 2008.

${ }^{9}$ D. E. Koditschek. Exact robot navigation by means of potential functions: Some topological considerations. In Proceedings of the 1987 International Conference on Robotics and Automation. IEEE, 1987.

${ }^{10} \mathrm{~W}$ H Kwon, A M Bruckstein, and T Kailath. Stabilizing State-Feedback Design via the Moving Horizon Method. IEEE, 1982.

${ }^{11}$ J. C. Latombe. Robot Motion Planning. Kluwer Academic Publishers, 1991.

${ }^{12}$ E.L. Lawler. The Traveling Salesman Problem: A Guided Tour of Combinatorial Optimization. Wiley, 1985.

${ }^{13} \mathrm{~T}$. Lozano-Perez. Automatic planning of manipulator transfer movements. IEEE Transactions on Systems, Man and Cybernetics, 11:681-698, 1981.

${ }^{14} \mathrm{~T}$. Lozano-Perez and M.A. Wesley. An algorithm for planning collision-free paths among polyhedral obstacles. Communications of the ACM, 22:681-698, 1979.

${ }^{15}$ D Q Mayne and H Michalska. Receeding Horion Control of Nonlinear Systems. IEEE Transactions of Automatic Control, 35(7), July 1990.

${ }^{16}$ Office of the Secretary of Defense. Unmanned aircraft systems roadmap, 2005-2030.

${ }^{17}$ John Osborne and Rolf Rysdyk. Waypoint guidance for small uavs in wind. In Proceedings of the AIAA Infotech@Aerospace Conference, Spet 2005.

${ }^{18}$ K Savla, F. Bullo, and E. Frazzoli. On Traveling Salesperson Problem for Dubins' Vehicle: Stochastic and Dynamic Environments. In Proceedings of the 44th IEEE Conference on Decision and Control and the European Control Conference 2005. IEEE, 2005.

${ }^{19}$ J. T. Schwartz and M. Sharir. On the piano mover's problem: I. the case of a two-dimensional rigid polygonal body moving admist polygonal barriers. Communications on Pure and Applied Mathematics, 36:345-398, 1983.

${ }^{20}$ Claude Shannon. A Mechanical Theory of Communication. The Bell System Technical Journal, 27:379-423, 623-656, 1948.

${ }^{21}$ H.A. Simon. Bounded Rationality. 1987. 


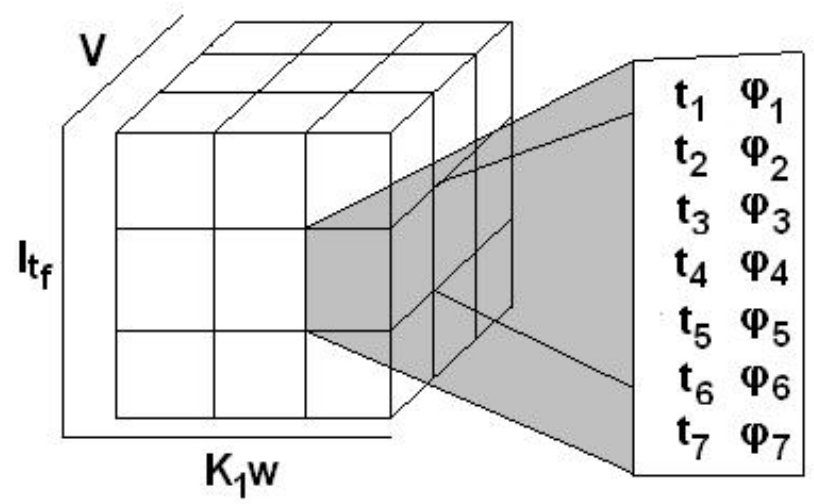

Figure 7. Visualization of an example lookup table with $r=3$.

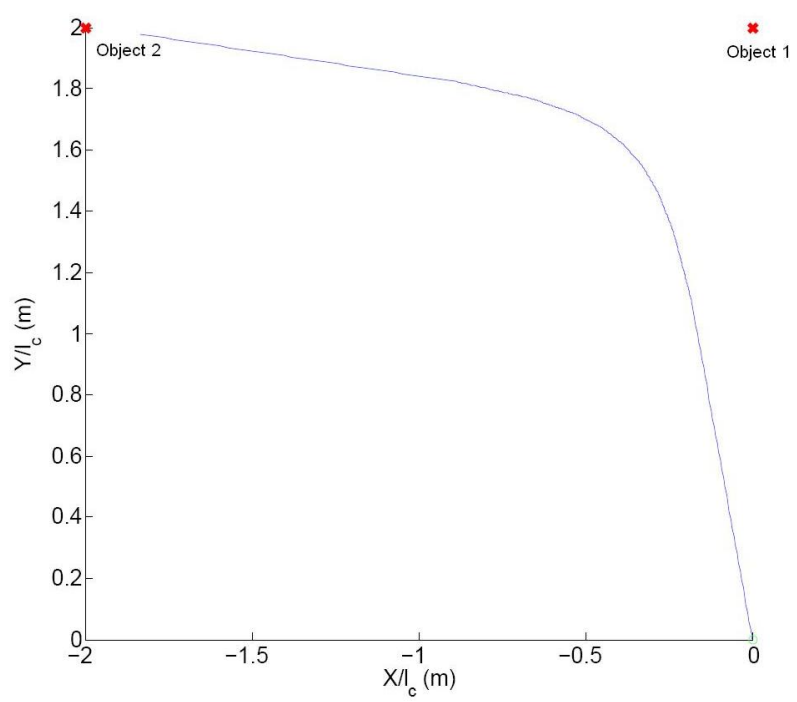

Figure 8. Optimal flight path for a single UAV using the discretization method

${ }^{22}$ P. Soueres and J. P. Laumond. Shortest paths synthesis for a car-like robot. IEEE Transactions on Automatic Control, pages 672-688, 1996.

${ }^{23} \mathrm{P}$. Svestka and M. Overmars. Coordinated motion planning for multiple car-like robots using probabilistic roadmaps. In Proceedings of the IEEE International Conference on Robotics and Automation. IEEE, 1995.

${ }^{24}$ S. Thrun, Y. Liu, D. Koller, A.Y. Ng, Z. Ghahramani, and H. Durrant-Whyte. Simultaneous Localization and Mapping with Sparse Extended Information Filters. The International Journal of Robotics Research, 23(7-8):693, 2004.

${ }^{25}$ S. Udupa. Collision detection and avoidance in computer controlled manipulators, 1977. 


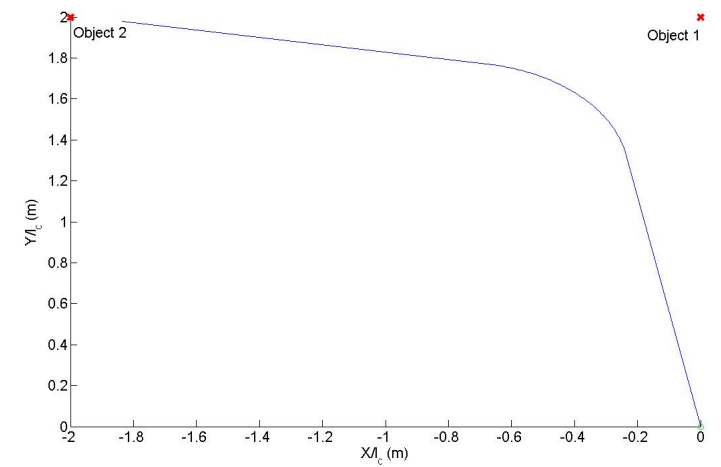

Figure 9. Near-optimal flight path for a single UAV using the parametric study strategy 